\title{
Indecisão profissional, ansiedade e depressão na adolescência: a influência dos estilos parentais
}

\author{
Claudio Simon Hutz. \\ Marúcia Patta Bardagir ${ }^{1}$
}

\begin{abstract}
Resumo
Este estudo investigou a influência dos estilos parentais percebidos sobre os níveis de indecisão profissional, ansiedade e depressão de adolescentes. Participaram do estudo 467 adolescentes, de 15 a 20 anos, estudantes do último ano do ensino médio em Porto Alegre. Os instrumentos utilizados foram um Questionário Sóciodemográfico, uma Escala de Indecisão Profissional, uma Escala de Estilos Parentais e os Inventários Beck de Ansiedade e Depressão. Houve correlação positiva entre indecisão, ansiedade e depressão. Filhos de pais autoritários e negligentes apresentaram maior depressão e ansiedade do que os outros. Os estilos parentais não influenciaram diretamente a indecisão profissional, mas sim o bem-estar psicológico dos adolescentes, indicando que o padrão de interação familiar é fundamental para o entendimento de como a indecisão está sendo vivenciada. Assim, enfatiza-se a importância do trabalho relativo à saúde emocional e à interação familiar nos processos de Orientação Profissional. Palavras-chave: Estilos parentais; Indecisão profissional; Bem-estar psicológico.
\end{abstract}

\section{Vocational indecision, anxiety and depression in adolescence: The influence of parenting styles}

\begin{abstract}
This study investigated perceived parenting styles and its influence on adolescents' vocational indecision, anxiety, and depression. Participants were 467 male and female high school students, aged 15 to 20 years old in Porto Alegre. Instruments were a demographic questionnaire, Beck Depression and Anxiety Inventories and scales to measure vocational indecision and perceived parenting styles. Positive correlation was found between indecision, anxiety, and depression. Adolescents from authoritarian and neglectful families scored significantly higher than others in depression and anxiety. Parenting styles had no direct influence on vocational indecision, but on adolescents' well being, suggesting that family interaction patterns are important to understand how indecision is been experienced. This study concludes that professional guidance processes must include family interaction aspects and must focus on adolescents' mental health.
\end{abstract}

Key words: Parenting styles; Career indecision; Psychological well being.

\section{Introdução}

A adolescência é uma fase de inúmeras mudanças biológicas, psicológicas e sociais, entre as quais estão a transformação das relações familiares e a definição da escolha profissional e preparação para o trabalho. Este estudo busca integrar os contextos familiar e vocacional identificando relações entre tipos de interação familiar percebida e o desenvolvimento vocacional dos adolescentes.

Freqüentemente, à medida que os filhos crescem, transferem seu investimento emocional dos pais em direção aos amigos (Rudolph \& Hammen, 1999), em busca de relações mais igualitárias e recíprocas entre os pares que contrastam com as relações hierárquicas na família (Young, Antal, Bassett, Post \& Valach, 1999). No entanto, pais são os outros privilegiados quando os assuntos são escola, mundo do trabalho e, especificamente, escolha profissional (Bednar \& Fisher, 2003; Destri, 1996; Magalhães, 1995), sendo citados como modelos, fontes de apoio à escolha e fontes de valores e expectativas profissionais. Aspectos como sexo (Alchieri \& Charczuk, 2002; Steele \& Barling, 1996) e idade (Alchieri \& Charczuk, 2002) costumam moderar a influência parental, apontando os meninos e os estudantes mais jovens como os mais suscetíveis. Ainda,

${ }^{1}$ Endereço para correspondência:

Rua Guilherme Alves, 450/204 - Bairro Jardim Botânico -90680-000 - Porto Alegre-RS

Tel: (51) 33165446

E-mail: marucia.bardagi@gmail.com 
indivíduos cujas famílias apresentam graus semelhantes de proximidade e autonomia seriam mais comprometidos e tranqüilos com a escolha, teriam maior ajustamento acadêmico, além de maior auto-eficácia para a tomada de decisão (Beyers \& Goossens, 2003; O’Brien, 1996). Dificuldades com a autonomia relacionam-se à indecisão ou ao comprometimento precipitado com escolhas profissionais, tanto pela dependência da opinião alheia como pelo baixo comportamento exploratório decorrente dessa pouca autonomia (Frischenbruder, 1999).

No Brasil, pesquisas apontam um papel mais diretivo dos pais, sugerindo certas profissões e sendo determinantes na escolha dos filhos (Cavalcante, Cavalcante \& Bock, 2001; Oliveira \& Dias, 2001). Oliveira e Dias (2001), em um estudo com mães de adolescentes diante do primeiro vestibular, demonstraram que elas consideram os filhos muito inseguros e imaturos para a escolha e assumem um papel mais controlador. Destri (1996) investigou expectativas de adolescentes sobre as participações ideais e reais de seus pais durante a escolha profissional; $55,8 \%$ dos jovens declararam que os pais deveriam apoiar a escolha e $27,4 \%$ que eles não deveriam ter nenhuma participação. Quanto à participação real dos pais, os adolescentes perceberam as mães como mais apoiadoras $(56 \%)$ do que os pais $(40,8 \%)$ e os pais foram mais percebidos como não tendo participação alguma na escolha $(41 \%)$ do que as mães $(28 \%)$.

É importante salientar que o papel da família não deve ser analisado isoladamente, pois há um contexto de escolha profissional e de carreira que engloba a situação educacional e econômica dos adolescentes (Kohn, 1995; Shek, 2005), as oportunidades percebidas e, no caso brasileiro, o próprio vestibular. Mas é na família que as negociações objetivas e subjetivas entre esses fatores irão se realizar. Embora as teorias do desenvolvimento de carreira salientem o papel da família para o desenvolvimento vocacional dos filhos, a natureza dessa influência ainda não é clara e permanece como um grande campo de investigação. No Brasil, especificamente, o papel familiar foi bastante explorado dentro de perspectivas dinâmicas de escolha profissional e é enfatizado nas propostas de intervenção com adolescentes, mas poucos estudos empíricos existem a fim de avaliar o impacto familiar global sobre o contexto da escolha profissional com base em uma perspectiva mais funcionalista, especialmente a partir da percepção dos filhos sobre as atitudes e comportamentos parentais.

Nesse sentido, uma das formas de análise das práticas parentais e suas influências, muito utilizada internacionalmente, mas pouco conhecida no Brasil, é a descrição dos estilos parentais, conjunto de atitudes, práticas e expressões que caracterizam as interações pais-filhos (Baumrind, 1967, 1971). Os estilos se referem, basicamente, às posições parentais perante problemas disciplinares, controle do comportamento, necessidades emocionais dos filhos e tomada de decisões. A descrição dos estilos parentais como padrões globais e razoavelmente estáveis de comportamento em relação aos filhos foi feita por Baumrind $(1967,1971)$ em sua tipologia das práticas de socialização. Ela descreveu três padrões de controle parental (autoritário, autoritativo e permissivo), e suas conseqüências sobre os filhos. Posteriormente, Maccoby e Martin (1983) reclassificaram os estilos a partir da exigência (controle, estabelecimento de padrões de conduta, disciplina) e responsividade (comunicação, afetividade, aquiescência) disponibilizadas pelos pais. Surgiram, então, quatro estilos: os padrões autoritário e autoritativo descritos por Baumrind (1967, 1971), e os novos padrões indulgente e negligente. Esta classificação é a mais utilizada nos estudos sobre o tema.

Em uma breve descrição (ver detalhes em Reppold, Pacheco, Bardagi \& Hutz, 2002), o estilo autoritário (exigência alta e responsividade baixa) é aquele cujos pais esperam obediência e usam mais a força, buscando a autoridade, sem encorajar o diálogo e a autonomia. Filhos criados sob este padrão costumam apresentar poucos problemas de comportamento e alto desempenho acadêmico (Lamborn, Mounts, Steinberg \& Dornbusch, 1991), mas menor auto-estima, medo e frustração, maior ansiedade e depressão (Aunola, Sttatin \& Nurmi, 2000; Reppold \& Hutz, 2003; Wolfradt, Hempel \& Miles, 2003) e insegurança nas interações sociais (Pacheco, Teixeira \& Gomes, 1999). No estilo autoritativo (authoritative $e_{2}$ no original), com exigência e responsividade altas, os pais dão razões para as restrições impostas e favorecem o diálogo, encorajando a autonomia e sendo responsivos. Crianças criadas neste padrão têm melhor desempenho do que aquelas criadas sob os outros padrões (Baumrind, 1971), como maior auto-estima (Jackson, Pratt, Hunsberger \& Pancer, 2005), motivação para realização, competência social e cognitiva e poucos problemas de internalização e comportamento (Adalbjarnardottir \& Hafsteinsson, 2001; Aunola \& cols., 2000; Lamborn \& cols., 1991; Wolfradt \& cols., 2003).

$\mathrm{O}$ estilo indulgente (exigência baixa e responsividade alta) caracteriza-se pela tolerância, pelo afeto e pelo baixo controle. Os pais são complacentes, raramente fazendo exigências ou aplicando punições. Filhos criados neste padrão costumam ter boa autoestima e bem-estar psicológico, mas maior imaturidade, pouco envolvimento escolar, agressividade e problemas de comportamento (Glasgow, Dornbusch, Troyer, 
Steinberg \& Ritter, 1997; Lamborn \& cols., 1991; Wolfradt \& cols., 2003). No estilo negligente (exigência e responsividade baixas) os pais são fracos em controlar o comportamento dos filhos e em atender suas necessidades e demonstrar afeto. Filhos criados sob este padrão apresentam os piores índices de ajustamento entre os quatro estilos, com menor competência social e cognitiva e mais problemas de internalização e comportamento (Adalbjarnardottir \& Hafsteinsson, 2001; Reppold \& Hutz, 2003).

Os pais costumam ser mais autoritários ou negligentes do que as mães, e elas mais autoritativas ou indulgentes (Conrade \& Ho, 2001; Russell, Aloa, Feder, Glover \& cols., 1998). Conrade e Ho (2001) salientam que meninos percebem pais como mais autoritários e mães como mais permissivas, enquanto meninas percebem mães como mais autoritativas. Quanto à prevalência, os estilos autoritativo e negligente costumam predominar, seguidos pelos estilos autoritário e indulgente (Costa, Teixeira, \& Gomes, 2000; Lamborn \& cols., 1991; Reppold \& Hutz, 2003; Pacheco \& cols., 1999; Weber, Prado, Viezzer \& Brandenburg, 2004). A percepção do comportamento parental também se altera com a idade, sendo que crianças tendem a ter uma visão mais positiva, e adolescentes uma visão mais negativa do comportamento dos pais (Shek, 1998).

Percebe-se, assim, a relação entre estilos parentais e características importantes para o desenvolvimento vocacional, como autonomia, autoestima e autoconfiança. No âmbito vocacional, a família é um aspecto fundamental para a escolha profissional dos filhos. Ao relacionar esses dois contextos, este estudo buscou investigar a relação entre os estilos parentais percebidos pelos adolescentes e seus níveis de indecisão, ansiedade e depressão. Tem-se a expectativa de que filhos criados sob o padrão autoritativo apresentem os menores índices nas medidas avaliadas, resultado oposto ao esperado para filhos criados sob o padrão negligente. Quanto ao estilo autoritário, pode estar associado à menor indecisão, mas também a maior ansiedade e depressão; já o estilo indulgente pode estar relacionado a maior indecisão, mas a menor ansiedade e depressão. Esperam-se índices mais elevados de indecisão, ansiedade e depressão nas meninas com base nos resultados de diversos estudos (La Rosa, 1998; Locker \& Cropley, 2004; Matos, Barrett, Dadds \& Shortt, 2003). No entanto, a literatura não tem indicado sistematicamente diferenças de sexo nos estudos relativos à indecisão (Neiva, 2003).

\section{Método}

Participantes: Participaram do estudo 467 estudantes gaúchos (52,9\% meninas) do terceiro ano do Ensino Médio entre 15 e 20 anos ( $M=17$ anos). A maioria estuda em escola privada (54\%), tem pais casados $(79,7 \%)$ e não trabalha $(89,3 \%)$.

Instrumentos: Além de um questionário sociodemográfico, foram utilizados os seguintes instrumentos (todos de auto-relato e do tipo Likert):

a) Escala de Estilos Parentais (Teixeira \& Gomes, 2000, manuscrito não publicado): instrumento elaborado com base na Escala de Responsividade e Exigência Parental (Lamborn \& cols., 1991; adaptada por Costa \& cols., 2000), com 15 itens relativos à exigência e 18 relativos à responsividade. Os participantes apontam a freqüência em que pai e mãe (separadamente) apresentam o comportamento descrito. São classificados os estilos paterno e materno e também o estilo combinado do casal. Os índices de consistência interna (alfa de Cronbach) foram 0,77 e 0,93 para as escalas de exigência e responsividade. Após este estudo, o instrumento foi refinado e resultou na escala de Responsividade e Exigência Parental (Teixeira, Bardagi \& Gomes, 2004).

b) Escala de Indecisão Profissional (Teixeira \& Gomes, 1999, manuscrito não publicado): Escala de 30 itens refletindo um índice geral de indecisão, com avaliação somatória, criada com base em instrumentos internacionais clássicos na área além de itens originais criados a partir da literatura. $\mathrm{O}$ instrumento mostrou consistência interna (alfa de Cronbach) de 0,93.

c) Inventários Beck de Depressão e Ansiedade (Cunha, 2001): Cada uma das escalas é composta por 21 itens, indicando desde graus mínimos até níveis graves de ansiedade e depressão. As avaliações são somatórias, e os índices de consistência interna (alfa de Cronbach) obtidos foram 0,82 para o BDI e 0,87 para o BAI.

Procedimento e Consideracões Éticas: Os participantes responderam aos instrumentos em aplicações coletivas em sala de aula, após explicação dos objetivos do estudo, e a participação foi voluntária. Houve autorização (Consentimento Livre e Esclarecido) dos pais, da escola e também dos adolescentes. Durante a coleta e análise de dados foram garantidos o sigilo e a confidencialidade dos mesmos. 
Tabela 1 - Resultados em ansiedade, indecisão e depressão por sexo, escola e estilo parental

\begin{tabular}{|c|c|c|c|c|c|c|c|c|}
\hline \multirow{4}{*}{ Sexo } & & \multirow[b]{2}{*}{$\mathrm{n}$} & \multicolumn{2}{|c|}{ Indecisão } & \multicolumn{2}{|c|}{ Ansiedade } & \multicolumn{2}{|c|}{ Depressão } \\
\hline & & & M & $d p$ & M & $\mathrm{dp}$ & M & $d p$ \\
\hline & Masc. & 220 & 72,3 & 14,11 & 7,3 & 6,30 & 8,0 & 6,97 \\
\hline & Fem. & 247 & 73,3 & 16,10 & 10,9 & 8,36 & 8,9 & 6,23 \\
\hline \multicolumn{9}{|c|}{ Escola } \\
\hline & Pública & 215 & 73,8 & 15,75 & 10,0 & 7,61 & 9,2 & 6,17 \\
\hline & Privada & 252 & 72,0 & 14,67 & 8,6 & 7,66 & 7,9 & 6,89 \\
\hline \multicolumn{9}{|c|}{ Est. Parental } \\
\hline & Autoritativo & 119 & 72,8 & 14,24 & 7,9 & 7,39 & 5,4 & 4,44 \\
\hline & Autoritário & 88 & 73,7 & 16,57 & 10,2 & 7,31 & 10,5 & 6,38 \\
\hline & Indulgente & 89 & 70,7 & 16,04 & 9,2 & 8,50 & 8,6 & 7,00 \\
\hline & Negligente & 119 & 72,8 & 14,18 & 9,7 & 7,27 & 10,0 & 7,36 \\
\hline
\end{tabular}

\section{Resultados}

As médias femininas foram mais altas do que as masculinas em indecisão, ansiedade e depressão. Estes resultados, separados por sexo, tipo de escola e estilo parental percebido, estão na Tabela 1. A maioria dos adolescentes $(63 \%)$ relata ter escolha profissional definida. Em relação à indecisão, os resultados apontam menores escores entre os adolescentes já definidos $(t=9,32 ; g l=455 ; p<0,01)$ e entre filhos únicos ou do meio em relação aos outros $[\mathrm{F}(3,452)=4,27 ; p<0,01]$. Não houve diferenças de sexo ou tipo de escola nos escores de indecisão. Em depressão, adolescentes com escores moderado e grave (considerados clinicamente deprimidos) somam 5,1\%. Alunos da rede pública têm escores maiores do que alunos da rede privada $(t=2,04$; $g l=465 ; p<0,05)$. Diferenças de sexo quanto ao escore total ou aos níveis de depressão não foram observadas. Em ansiedade, adolescentes clinicamente ansiosos somam $9 \%$ da amostra. Meninas apresentam maior ansiedade do que meninos $(t=5,17 ; g l=465 ; p<0,01)$ e alunos da rede pública têm maior ansiedade do que os da rede privada $(t=2,02 ; g l=465 ; p<0,05)$.

As médias paternas e maternas em responsividade e exigência de acordo com meninos e meninas estão na Tabela 2. As mães apresentam médias maiores nas duas dimensões. As meninas percebem maior responsividade $(t=3,17 ; g l=465 ; \mathrm{p}<0,01)$ e exigência $(t=4,76$; $g \underline{l}=465 ; p<0,01)$ maternas do que os meninos. Diferenças na percepção de exigência e responsividade paternas não foram observadas, assim como diferenças na percepção do comportamento parental entre os dois tipos de escola.

Ansiedade $(\mathrm{r}=0,32)$ e depressão $(r=0,32)$ são as medidas com maior correlação com indecisão profissional. A depressão se correlaciona negativamente também com responsividade $(r=-0,32)$ e exigência $(r=-$ $0,12)$ parental. Ansiedade e depressão mostram correlação positiva $(r=0,50)$, assim como exigência e responsividade $(\mathrm{r}=0,22)$.

$\mathrm{Na}$ descrição dos estilos parentais, a Figura 1 mostra os percentuais válidos dos estilos materno,

Tabela 2 - Resultados em exigência e responsividade parentais

\begin{tabular}{lcccc}
\hline & \multicolumn{2}{c}{ Meninas } & \multicolumn{2}{c}{ Meninos } \\
\cline { 2 - 5 } Exigência & $\mathrm{M}$ & $\mathrm{dp}$ & $\mathrm{M}$ & $\mathrm{dp}$ \\
Pais & & & & \\
Mães & 26,8 & 10,42 & 25,1 & 8,48 \\
Combinada & 30,2 & 10,08 & 26,2 & 7,37 \\
& 57,2 & 17,90 & 51,5 & 14,66 \\
\hline Responsividade & & & & \\
Pais & & & 47,6 & 13,87 \\
Mães & 47,0 & 16,68 & 51,8 & 11,67 \\
Combinada & 55,4 & 13,15 & 99,2 & 23,64 \\
& 101,7 & 25,75 & & \\
\hline
\end{tabular}




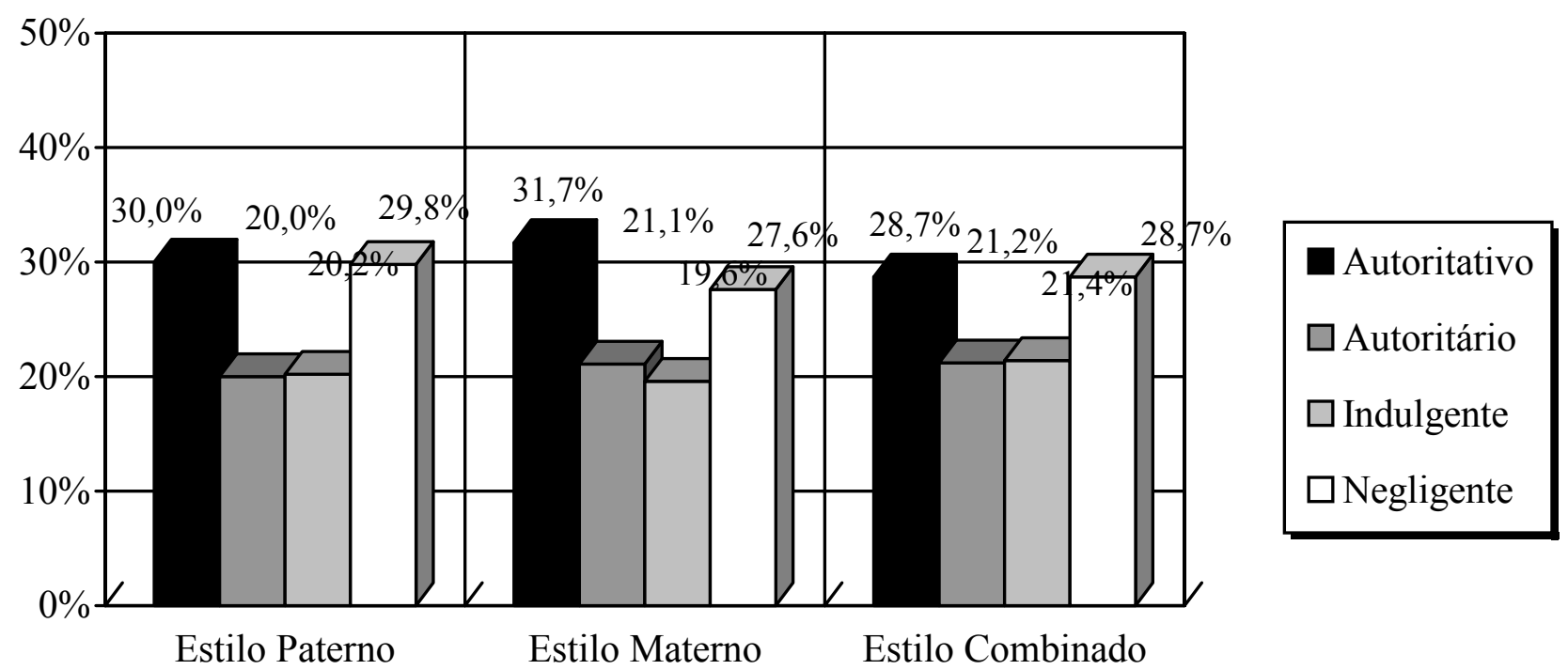

Figura 1 - Freqüência dos estilos parentais paterno, materno e combinado

paterno e combinado. Há predomínio do estilo autoritativo tanto para pais quanto para mães. As mães foram mais freqüentemente descritas como autoritativas $(31,7 \%)$, negligentes $(27,6 \%)$, autoritárias $(21,1 \%)$ e indulgentes $(19,6 \%)$; os pais foram mais freqüentemente descritos como autoritativos (30\%), negligentes $(29,8 \%)$, indulgentes $(20,2 \%)$ e autoritários $(20 \%)$. Ao tomar meninos e meninas em separado, há um aumento, na visão dos meninos, da negligência e uma diminuição da autoritatividade maternas $\left(\chi^{2}=36,72\right.$; $g l=3$; $p<0,01)$. Diferenças nos estilos paterno e materno na escola pública não são observadas.

Ao analisarmos os níveis de indecisão, ansiedade e depressão por tipo de interação familiar (Tabela 1), vemos que filhos de pais autoritativos apresentam menor depressão do que filhos de pais autoritários, indulgentes e negligentes $(t=-6,64 ; g l=205$; $p<0,01 ; \quad t=-3,98 ; \quad g l=206 ; \quad p<0,01 ; \quad t=-5,79 ; \quad g l=236$; $p<0,01)$, e menor ansiedade do que filhos de pais autoritários $(t=-2,20 ; g l=205 ; p<0,05)$ e negligentes $(t=$ $-1,90 ; g l=236 ; p<0,05)$. Em indecisão, não há diferenças entre os grupos. Filhos de pais autoritários e negligentes não diferem entre si em ansiedade ou depressão. Estes resultados confirmam parcialmente as hipóteses iniciais do estudo.

\section{Discussão}

Os resultados obtidos apontam várias consistências em relação à literatura. Os índices de ansiedade e depressão mostram-se próximos aos encontrados em outros estudos (Reppold \& Hutz, 2003), assim como as diferenças de gênero e as correlações entre indecisão e medidas emocionais (Frischenbruder, 1999; Jones \& Winer, 1991).

As médias femininas mais elevadas em indecisão, ansiedade e depressão são compatíveis com os resultados encontrados na literatura (La Rosa, 1998; Locker \& Cropley, 2004; Matos e cols., 2003). No entanto, deve-se salientar que esses escores mais altos em avaliações subjetivas são comuns e podem estar refletindo um maior cuidado e atenção das meninas aos próprios estados internos (maior preocupação com o autoconhecimento) e não necessariamente mais problemas de internalização ou neuroticismo. Os adolescentes mais indecisos mostram-se também mais deprimidos e ansiosos, indicando que a dificuldade em realizar a opção profissional (especialmente quando isso é socialmente solicitado) prejudica o bem-estar. A menor indecisão entre adolescentes que declaram já possuírem uma escolha sugere que estas opções são confiáveis, congruentes com seus interesses e preferências vocacionais e não estariam apenas respondendo às demandas sociais ou encobrindo outros conflitos. Por outro lado, quem ainda não está definido apresenta maior depressão, confirmando que à medida que o tempo passa quem não escolheu vivencia sensações maiores de desvalia, incompetência, além da desaprovação social.

As relações entre tipo de escola e índices de ansiedade e depressão apontam menor bem-estar psicológico dos alunos da rede pública. $O$ fator econômico pode ser um mediador dessa relação, uma vez que há evidências de que adolescentes de nível socioeconômico baixo têm menor bem-estar (Kohn, 1995; Shek, 2005). No entanto, a própria questão 
profissional pode desencadear piores índices de ajustamento, já que esses adolescentes costumam se sentir menos capazes de atingir os objetivos e metas profissionais do que alunos da rede privada, bem como menos competitivos em relação ao vestibular, por exemplo.

$\mathrm{Na}$ indecisão, os escores não apresentam, na maioria dos aspectos analisados, variações significativas entre os alunos. A indecisão aparece, dessa forma, como um componente esperado, inerente ao processo de escolha vocacional, que deve ser encarada como um fator normativo e até positivo, pois permite a reflexão. A ausência de diferenças nos níveis de indecisão profissional entre alunos de escolas públicas e privadas mostra que as possibilidades de acesso à informação profissional podem estar mais uniformemente disponíveis e outros fatores como o grande número de opções e a própria precocidade da escolha podem fazer com que as eventuais dificuldades em estabelecer uma preferência estejam afetando a esses adolescentes de forma semelhante. A maior diferença entre os alunos das redes pública e privada não é a capacidade de escolher ou não uma opção profissional, mas a forma como eles se sentem a respeito das próprias escolhas, como apontaram os índices de ansiedade e depressão. Quanto à semelhança dos índices de indecisão entre meninas e meninos, esse resultado é coerente com outros achados (Neiva, 2003).

Já a posição na família pode levar a situações diferentes no momento da escolha. Filhos únicos e do meio apresentam maior indecisão do que os outros. Os primeiros podem estar inaugurando na família as preocupações profissionais e o contato com o vestibular, gerando nos outros, e também em si mesmos, muita expectativa. Já os filhos do meio, embora sua maior indecisão seja surpreendente (pela ausência de achados semelhantes anteriores), podem estar reagindo a uma comparação com os irmãos mais velhos, sendo pressionados a superá-los se estes fracassaram ou a igualar-se a eles se foram bem sucedidos em sua escolha. Em qualquer caso, a participação da família é fundamental para a compreensão do processo decisório.

$\mathrm{Na}$ descrição do comportamento parental, um aspecto a ser destacado é a relevância da presença materna, especialmente para as meninas, confirmando a maior proximidade da mãe com os filhos, apontada na literatura (Baumrind, 1967, 1971; Costa \& cols., 2000; Paulson \& Sputa, 1996). De forma geral, as meninas percebem maiores níveis de exigência e responsividade por parte da mãe do que os meninos e têm uma tendência a descrever também o pai como mais exigente. Esses dados são congruentes com pesquisas anteriores apontando que os pais (especialmente as mães) tendem a ser mais responsivos com as meninas e que as meninas são mais sensíveis na percepção e descrição das interações familiares (Conrade \& Ho, 2001; Costa \& cols., 2000; Paulson \& Sputa, 1996; Russell \& cols., 1998). Uma pesquisa com crianças aponta diferenças apenas na dimensão exigência (Weber \& cols., 2004), o que indica que talvez à medida que os filhos crescem os pais tendem a também disponibilizar afeto e atenção diferencialmente. As diferenças no estilo materno apontam para eventuais dificuldades que as mães estariam encontrando para lidar com os filhos homens na adolescência, especialmente em relação ao fornecimento de afeto. Essas dificuldades acarretam menor envolvimento materno em geral, caracterizando as mães como mais negligentes na percepção dos meninos.

A descrição dos estilos parentais segue a distribuição encontrada em estudos internacionais e nacionais anteriores (Costa \& cols., 2000; Lamborn \& cols., 1991; Pacheco \& cols., 1999; Weber \& cols., 2004). Há predomínio dos estilos autoritativo e negligente, seguidos pelos estilos indulgente e autoritário. $\mathrm{O}$ alto índice de autoritatividade deve ser visto de forma positiva, pois demonstra que muitos casais estão conseguindo equilibrar práticas de controle e disciplina com afetividade e respeito pelos filhos. Por outro lado, a alta negligência é preocupante e mostra que existem muitos pais incapazes de cumprir suas funções de monitorar e proteger os filhos, oferecendo-lhes, ao mesmo tempo, limites e afeto. $O$ alto índice de negligência também nas escolas privadas deixa claro que a falta de comprometimento de alguns pais não é somente uma questão de condições socioeconômicas ou baixa escolaridade, mas uma condição que atinge todas as camadas sociais entre as famílias modernas.

É possível pensar, no entanto, que a negligência não seja intencional em todos os casais, mas em muitos casos fruto de um desencontro entre as expectativas e práticas parentais e a interpretação feita pelos filhos. Além disso, é preciso considerar que os filhos tendem a apresentar uma visão mais negativa das interações familiares na adolescência (Shek, 1998). Nesse sentido, torna-se fundamental que haja maior comunicação familiar (Weber \& cols., 2004), que os pais possam explicitar aos filhos as razões de seu comportamento e que os filhos possam apontar a percepção que têm das práticas parentais. Explicitamente, Young e colaboradores (1999) indicaram a discussão aberta entre pais e filhos sobre os projetos de carreira e trabalho como uma forma de diminuir a ansiedade e conhecer as expectativas de familiares.

As relações entre indecisão, ansiedade $\mathrm{e}$ depressão e as interações familiares permitem a discriminação de diferentes contextos de escolha. E essas 
relações permitem corroborar o papel fundamental que os pais exercem no âmbito vocacional, apontado na literatura (Bednar \& Fisher, 2003; Destri, 1996; Magalhães, 1995). O índice de indecisão entre os filhos de pais autoritativos, por exemplo, pode estar refletindo dificuldades objetivas de escolha, mas não prejuízo ao bem-estar psicológico, como mostram os baixos escores em ansiedade e depressão. Dessa forma, podemos pensar que indecisão profissional quando não acompanhada de ansiedade ou depressão pode ser vista de uma forma positiva, um reflexo de maior exploração de carreira, especialmente em adolescentes mais jovens. Esses adolescentes podem estar se permitindo maior reflexão sobre a carreira, ou mesmo a postergação da escolha e a elaboração de outros planos mais imediatos, sem pressão familiar ou imposição de alternativas profissionais. Beyers e Goossens (2003) e O'Brien (1996) já haviam apontado uma maior maturidade e tranqüilidade para a escolha profissional em famílias com graus semelhantes de proximidade e autonomia, características típicas de famílias autoritativas. Pais autoritativos podem estar ajudando o adolescente a vivenciar a indecisão como um estado normal e esperado neste momento, preservando a auto-estima dos filhos (Jackson \& cols., 2005; Krumboltz, 1992).

Já filhos de pais negligentes e autoritários, por seus escores em ansiedade e depressão, estariam sob um grau elevado de sofrimento psicológico e podem apresentar dificuldades em outros contextos além da escolha profissional, como problemas de interação social, dificuldades escolares, entre outros (Adalbjarnardottir \& Hafsteinsson, 2001; Pacheco \& cols., 1999; Reppold \& Hutz, 2003). Pais autoritários tendem a não permitir dúvida ou hesitação na hora da escolha, exigindo a tomada de decisão por parte do adolescente, ou mesmo criticando ou desacreditando eventuais decisões tomadas. Como no Brasil há uma certa tendência à maior diretividade parental (Cavalcante \& cols., 2001; Oliveira \& Dias, 2001) na escolha dos filhos, isso pode ser percebido pelos adolescentes como autoritarismo e gerar um desconforto emocional maior. Por sua vez, o desengajamento dos pais negligentes não possibilita ao adolescente estabelecer sentimentos de confiança ou segurança em sua própria capacidade de escolha. Além disso, pais negligentes não costumam oferecer disponibilidade para troca de informações ou servir de modelos para os filhos. Aunola e colaboradores (2000) ressaltam o fato de pais autoritativos e indulgentes encorajarem a exploração e auto-regulação e confiarem na capacidade dos filhos, criando uma atmosfera de aceitação. Por outro lado, o envolvimento familiar negativo típico de famílias autoritárias e negligentes, é fonte de desajustamento e dificuldades de escolha.

Concluindo, pode-se dizer que os estilos parentais afetam o desenvolvimento dos filhos de forma global, na formação de competências básicas que gradativamente influenciam comportamentos complexos como a decisão profissional. Por isso, as interações familiares tornam-se ainda mais importantes na compreensão das situações vivenciadas pelos filhos na adolescência, sendo um referencial de análise fundamental também em etapas posteriores da socialização (Baumrind, 1967, 1971). As teorias do desenvolvimento vocacional precisam enfatizar a influência parental, não apenas incluindo a participação dos pais nos programas de intervenção, mas analisando o clima familiar através da percepção dos adolescentes. Os resultados relativos ao bem-estar psicológico no momento da escolha tornam imprescindíveis que as intervenções em Orientação Profissional enfoquem ainda a saúde emocional dos adolescentes (Destri, 1996; Frischenbruder, 1999). A maioria dos processos atuais prioriza aspectos cognitivos em detrimento dos aspectos emocionais. É preciso apontar, no entanto, algumas ressalvas. Primeiramente, a utilização de um delineamento transversal, embora permita a descrição de relações importantes entre a variáveis analisadas, não possibilita a observação dessas relações ao longo do tempo. Em futuras investigações, metodologias qualitativas e estudos longitudinais podem auxiliar na compreensão de outros aspectos da influência parental sobre o desenvolvimento vocacional dos filhos.

\section{Referências}

Adalbjarnardottir, S. \& Hafsteinsson, L. G. (2001). Adolescents' perceived parenting styles and their substance use: Concurrent and longitudinal analyses. Journal of Research on Adolescence, 11(4), 401-423.

Alchieri, J. C. \& Charczuk, S. B. (2002). Escolha vocacional: aspectos da tomada de decisão em vestibulandos. Aletheia, 15(1), 7-14

Aunola, K., Sttatin, H. \& Nurmi, J. (2000). Parenting styles and adolescents' achievement strategies. Journal of Adolescence, 23(2), 205-222.

Baumrind, D. (1967). Child care practices anteceding three patterns of preschool behavior. Genetic Psychology Monographs, 75, 43-88.

Baumrind, D. (1971). Current patterns of parental authority. Developmental Psychology Monographs, 4, 1-103. 
Bednar, D. E. \& Fisher, T. D. (2003). Peer referencing in adolescent decision maeing as a function of perceived parenting style. Adolescence, 38, 607-621

Beyers, W. \& Goossens, L. (2003). Psychological separation and adjustment to university: Moderating effects of gender, age, and perceived parenting style. Journal of Adolescent Research, 18, 363-382.

Cavalcante, A. C. S., Cavalcante, R. \& Bock, S. (2001). Orientação profissional para estudantes de ensino médio em Teresina-PI. Anais do II Congresso Norte Nordeste de Psicologia, Salvador, (ESCPN 275).

Conrade, G. \& Ho, R. (2001). Differential parenting styles for fathers and mothers: Differential treatment for sons and daughters. Australian Journal of Psychology, 53(1), 29-35.

Costa, F. T., Teixeira, M. A. P. \& Gomes, W. B. (2000). Responsividade e exigência: Duas escalas para avaliar estilos parentais. Psicologia: Reflexão e Crítica, 13(3), 465-473.

Cunha, J. A. (2001). Escalas Beck. São Paulo: Casa do Psicólogo.

Destri, F. S. (1996). Relações entre pais e filhos adolescentes e o processo de escolha profissional (Dissertação de Mestrado). Porto Alegre: Universidade Federal do Rio Grande do Sul - Curso de Pós-Graduação em Psicologia do Desenvolvimento.

Frischenbruder, S. L. (1999). O desenvolvimento vocacional na adolescência: autoconceito e comportamento exploratório (Dissertação de Mestrado). Porto Alegre: Pontifícia Universidade Católica do Rio Grande do Sul Programa de Pós-Graduação em Psicologia Clínica.

Glasgow, K. L., Dornbusch, S. M., Troyer, L., Steinberg, L. \& Ritter, P. L. (1997). Parenting styles, adolecents' attributions, and educational outcomes in nine heterogeneous high schools. Child Development, $68,507-529$.

Jackson, 1. M., Pratt, M. W., Hunsberger, B. \& Pancer, S. M. (2005). Optimism as a mediator of the relation between perceived parental authoritativeness and adjustment among adolescents: Finding the sunny side of the street. Social Development, 14(2), 273-304.

Jones, K. T. \& Winer, J. L. (1991). Anxiety reduction in vocationally undecided students. Journal of Counseling and Human Service Professions, 20, 42-48.

Kohn, M. L. (1995). Social structure and personality through time and space. Em P. Moen, G. H. Elder Jr, \& K. Lüscher (Orgs.). Examining lives in context: Perspectives on the ecology of human development (pp. 141-
168). Washington: American Psychological Association.

Krumboltz, J. D. (1992). The wisdom of indecision. Journal of Vocational Behavior, 41, 239-244.

La Rosa, J. (1998). Ansiedade, sexo, nível socioeconômico e ordem de nascimento. Psicologia: Reflexão $e$ Crítica, 11(1), 59-70.

Lamborn, S. D., Mounts, N. S., Steinberg, L. \& Dornbusch, S. M. (1991). Patterns of competence and adjustment among adolescents from authoritative, authoritarian, indulgent, and neglectful families. Child Development, 62, 1049-1065.

Locker, J. \& Cropley, M. (2004). Anxiety, depression and self-esteem in secondary school children: An investigation into the impact of standard assessment tests (SATs) and other important school examinations. School Psychology International, 25(3), 333-345.

Maccoby, E. E. \& Martin, J. A. (1983). Socialization in the context of the family: Parent-child interaction. New York: Wiley.

Magalhães, M. O. (1995). Perspectiva experiencial da indecisão vocacional em adolescentes (Dissertação de Mestrado). Porto Alegre: Universidade Federal do Rio Grande do Sul - Curso de Pós-Graduação em Psicologia do Desenvolvimento.

Matos, M. G., Barrett, P., Dadds, M. \& Shortt, A. (2003). Anxiety, depression, and peer relationships during adolescence: Results from the Portuguese national health behaviour in school-aged children survey. European Journal of Psychology of Education, 17(1), 3-14.

Neiva, K. M. C. (2003). A maturidade para a escolha profissional: uma comparação entre alunos do ensino médio. Revista Brasileira de Orientação Profissional, 4, $97-$ 103.

O'Brien, K. M. (1996). The influence of psychological separation and parental attachment on the career development of adolescent women. Journal of Vocational Behavior, 48, 257-274.

Oliveira, I. D. \& Dias, C. M. S. B. (2001). De quem é o vestibular? Mãe frente ao processo de diferenciação do filho. Anais do II Congresso Norte Nordeste de Psicologia, Salvador, (FAMPN 488).

Pacheco, J. T. B., Teixeira, M. A. P. \& Gomes, W. B. (1999). Estilos parentais e desenvolvimento de habilidades sociais na adolescência. Psicologia: Teoria e Pesquisa, 15(2), 117-126. 
Paulson, S. E. \& Sputa, C. L. (1996). Patterns of parenting during adolescence: Perceptions of adolescents and parents. Adolescence, 31(122), 369-381.

Reppold, C. T. \& Hutz, C. S. (2003). Exigência e responsividade parental como preditores de depressão em adolescentes no sul do Brasil. Avaliação Psicológica, 3, 175-184.

Reppold, C. T., Pacheco, J. T. B., Bardagi, M. P. \& Hutz, C. S. (2002). Prevenção de problemas de comportamento e desenvolvimento de competências psicossociais em crianças e adolescentes: uma análise das práticas educativas e dos estilos parentais. Em: C. S. Hutz (Org.). Situações de risco e vulnerabilidade na infância e adolescência: aspectos teóricos e estratégias de intervenção (pp. 7-51). São Paulo: Casa do Psicólogo.

Rudolph, K. D. \& Hammen, C. (1999). Age and gender as determinants of stress exposure, generation, and reactions in youngsters: A transactional perspective. Child Development, 70, 660-677.

Russell, A., Aloa, V., Feder, T., Glover, A., Miller, H. \& Palmer, G. (1998). Sex-based differences in parenting styles in a sample with preschool children. Australian Journal of Psychology, 50(2), 89-99.

Shek, D. T. L. (2005). Perceived parental control processes, parent-child relational qualities, and psychological well-being in Chinese adolescents with and without economic disadvantage. Journal of Genetic Psychology, 166, 171-188.

Shek, D. T. (1998). A longitudinal study of Hong Kong adolescents' and parents' perceptions of family functionig and well-being. The Journal of Genetic Psychology, 159(4), 389-403.
Steele, J. \& Barling, J. (1996). Influence of maternal gender-role beliefs and role satisfaction on daughters' vocational interests. Sex Roles, 34(9/10), 637- 648.

Teixeira, M. A. P., Bardagi, M. P. \& Gomes, W. B. (2004). Refinamento de um instrumento para avaliar responsividade e exigência parental percebidas na adolescência. Avaliação Psicológica, 4, 1-12.

Teixeira, M. A. P. \& Gomes, W. B. (1999). Análise preliminar dos itens de uma escala para medir indecisão profissional. Manuscrito não-publicado.

Teixeira, M. A. P. \& Gomes, W. B. (2000). Escala revisada para avaliar atitudes parentais. Manuscrito nãopublicado.

Young, R. A., Antal, S., Bassett, M. E., Post, N. D. \& Valach, L. (1999). The joint actions of adolescents in peer conversations about career. Journal of Adolescence, 22(4), 527-538.

Weber, L. N. D., Prado, P. M., Viezzer, A. P. \& Brandenburg, O. J. (2004). Identificação de estilos parentais: O ponto de vista dos pais e dos filhos. Psicologia: Reflexão e Crítica, 17(4), 323-331.

Wolfradt, U., Hempel, S. \& Miles, J. N. V. (2003). Perceived parenting styles, depersonalisation, anxiety and coping behaviour in adolescents. Personality and Individual Differences, 34, 521-532

Recebido em agosto de 2005 Reformulado em outubro de 2005 Aprovado em fevereiro de 2006

Sobre os autores:

Claudio Simon Hutz é psicólogo, doutor em Psicologia pela Universidade de Iowa (EUA), professor titular do Instituto de Psicologia da UFRGS e coordenador do Laboratório de Mensuração. Foi presidente da ANPEPP e do IBAP.

Marúcia Patta Bardagi é psicóloga, mestre e doutoranda em Psicologia do Desenvolvimento pelo Programa de Pós-Graduação em Psicologia da UFRGS, docente dos cursos de Especialização em Avaliação Psicológica e Orientação Profissional da UFRGS e professora do curso de Psicologia da ULBRA/Santa Maria -RS. 
